\title{
COLLIMATION SYSTEMS FOR A TEV LINEAR COLLIDER*
}

\author{
N. MERMINGA, J. IRWIN, R. HELM and R.D. RUTH \\ Stanford Linear Accelerator Center, Stanford University, \\ Stanford, CA 94309, USA
}

(Received 3 August 1992; in final form 13 June 1994)

\begin{abstract}
Experience with the SLAC Linear Collider (SLC) has indicated that backgrounds caused by the transverse and energy tails of the beam distribution will be a serious problem for a next-generation linear collider. At small emittances mechanical scrapers do not provide a solution, because they can be damaged by the tiny, intense beams, and also because they induce wakefield kicks which cause emittance dilution.

In this paper, we present a possible solution which uses several nonlinear lenses to drive beam tails to large amplitudes where they can be more easily scraped mechanically. We present a design for transverse and energy collimation and study the optimization of such systems including wakefield effects and optical aberrations. Protection and design of the scrapers are discussed.
\end{abstract}

KEY WORDS: Beam transport, particle dynamics

\section{INTRODUCTION}

Experience with the SLAC Linear Collider (SLC) has indicated that backgrounds caused by transverse and energy tails of the beam distribution will be a fundamental problem of next-generation linear colliders. Even one high energy particle hitting the wall in the final quad can create unacceptable detector background.

Any collimation design for the next-generation linear colliders must satisfy the following requirements:

(1) It must provide an effective scraping despite the small (of the order of a micron) beam sizes. In Figure 1 we show the layout for a typical final doublet for X-Band beam parameters. The beam energy is $250 \mathrm{GeV}$. The magnet aperture is dictated to be about $2 \mathrm{~mm}$ from wakefield considerations, allowing for $1 \sigma^{a}$ beam jitter. With a pole tip field of 1.2 Tesla and a free length to the interaction point (IP) of 1meter, the geometry is uniquely determined. The plot for $500 \mathrm{GeV}$ is quite similar.

\footnotetext{
* Work supported by Department of Energy contract DE-AC03-76SF00515.

${ }^{a}$ The definitions of some basic accelerator physics concepts that we will encounter frequently in this work are reviewed in Appendix A.
} 


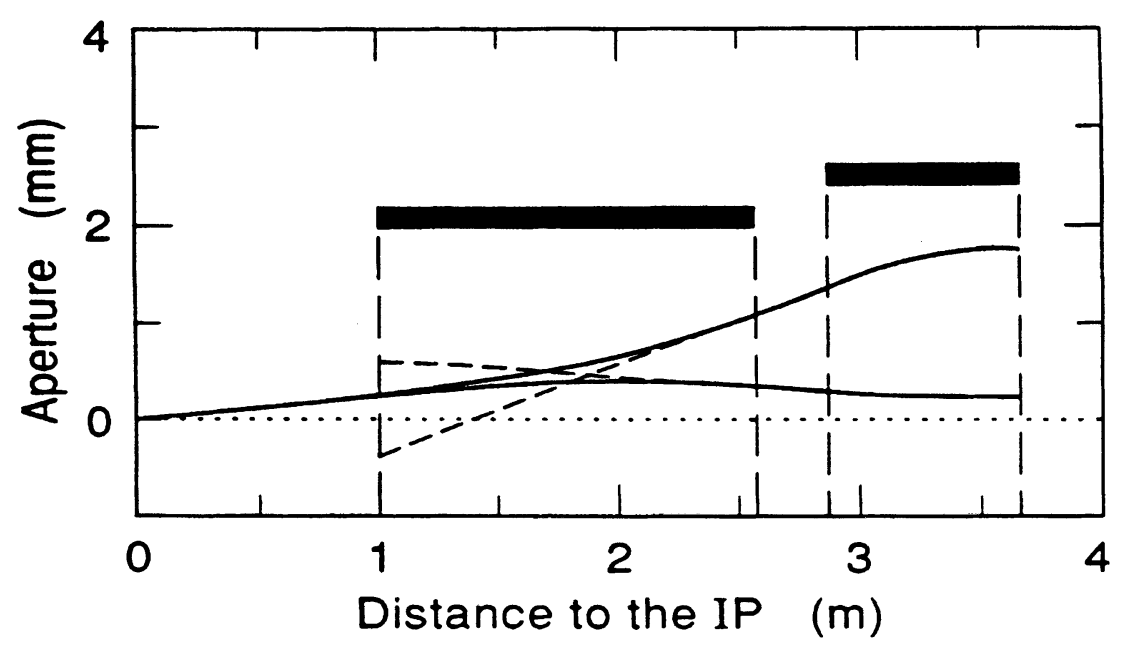

FIGURE 1: Layout of a typical final doublet for X-Band beam parameters.

The curved lines are beam particle trajectories at $10 \sigma_{x}$ and $10 \sigma_{y}$. The straight lines tangent to these curves indicate the synchrotron radiation envelope of these rays. For satisfactory operation, we assume that no beam particles and only a few synchrotron photons may hit the inner bore of the magnet.

Allowing some safety margin, we conclude that the $x$ motion must be collimated at about $5 \sigma_{x}$ and the $y$ motion at $15 \sigma_{y}$. Though our analysis will be for general " $n$ " sigma collimation, the specific lattice we construct will illustrate that it is possible to collimate at $5 \sigma$ in both $x$ and $y$ planes.

(2) It must protect scrapers against mis-steered beams which may hit them and possibly damage them. There are two problems associated with a train of ten bunches of $10^{10}$ electrons per bunch at $250 \mathrm{GeV}$ hitting a scraper. ${ }^{1}$ The first problem occurs at the surface of the scraper which may melt because of energy deposited in a small area. More quantitatively, we are interested in the largest spot size to cause failure of the scraper surface. For Ti, which is one of the best candidates according to SLC experience, the onset of melting at the surface occurs when the rms cross-sectional beam area is ${ }^{1}$

$$
\sigma_{x} \sigma_{y} \simeq 900 \mu \mathrm{m}^{2}
$$

The second problem occurs within the body of the scraper where the energy deposition from the shower peaks typically at several radiation lengths $\left(X_{0}\right)\left(\simeq 8 X_{0}\right.$ for $\left.\mathrm{Ti}\right)$.

(3) It must keep scraper-induced wakefield kicks on the beam below a tolerable level. If the beam does not pass exactly through the middle of the scrapers, it gets transverse deflections due to geometric and resistive wall wakefields. If these kicks are comparable to the angular divergence of the beam, the emittance will increase. 


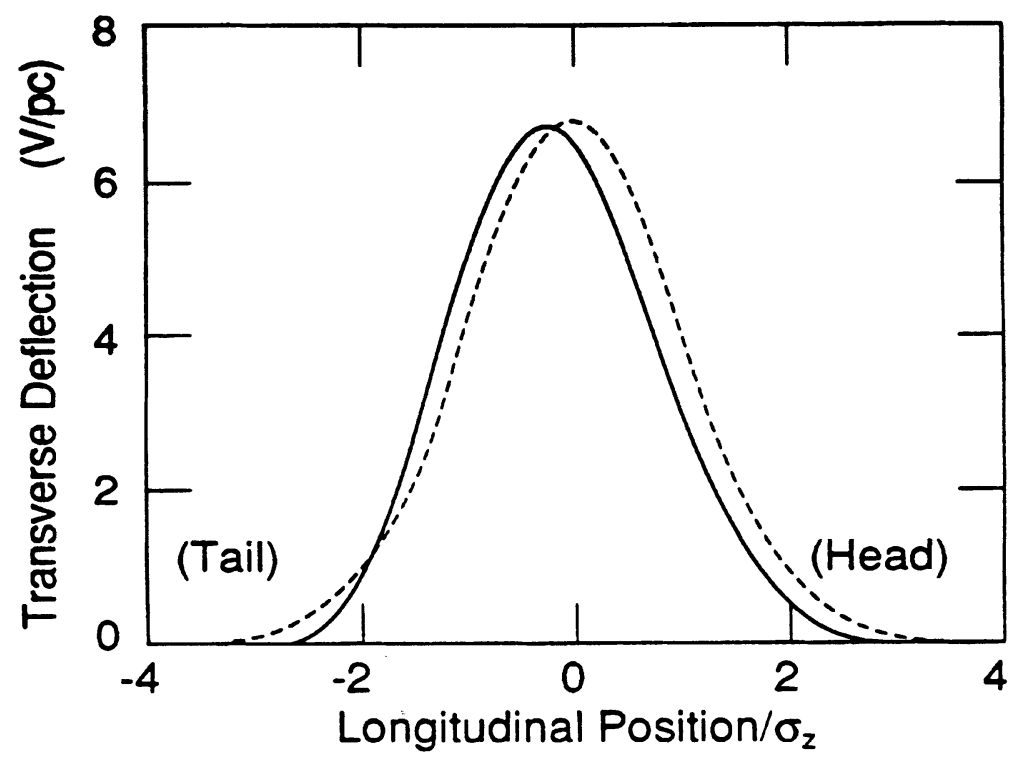

FIGURE 2: Kick of one scraper edge for beam size $\sigma_{z}=1.0 \mathrm{~mm}$ and scraper gap $g=0.5 \mathrm{~mm}$. The dotted line is the charge distribution.

The kick due to geometric wakefields varies longitudinally as the bunch density along the bunch, ${ }^{2}$ as shown in Figure 2. An expression for this kick, which includes the effect of both edges of a scraper, has been derived analytically and verified numerically ${ }^{2}$ under the assumption that the scraper gap is small compared to the scraper length, and the bunch length $\sigma_{z}$ is comparable to or longer than the scraper gap. For small offsets the transverse deflection of a particle is produced by the dipole wakefield only, and hence it is proportional to $\Delta\langle y\rangle / g$ where $\Delta\langle y\rangle$ is the beam offset from the middle of the scrapers and $2 g$ is the scraper gap. The wakefield kick, which is valid for cylindrically symmetric geometry, is given approximately by

$$
\Delta y^{\prime}(z)=\frac{4 r_{e} N}{\gamma} \frac{\Delta\langle y\rangle}{g} \frac{1}{\sqrt{2 \pi} \sigma_{z}} e^{-\frac{z^{2}}{2 \sigma_{z}^{2}}}
$$

where $\gamma=E / m c^{2}, N$ and $E$ are the number of particles per bunch and energy respectively, $z$ is the longitudinal position in the bunch and $r_{e}$ the classical radius of the electron. If the scraper consists of parallel plates separated by $2 g$, the kick is larger by a factor of $\pi^{2} / 8$. To reduce the effect of the geometric wakefield kick, one can taper the scrapers with a taper angle $\theta_{\text {tap }}\left(\theta_{\text {tap }}=\pi / 2\right.$ for a step scraper.) For small taper angles $\left(\theta_{\text {tap }} \leq 100 \mathrm{mrad}\right)$ the dependence on the taper angle is linear, ${ }^{3,4}$

$$
\Delta y^{\prime}(z)=\frac{\theta_{\mathrm{tap}}}{(\pi / 6)} \frac{4 r_{e} N}{\gamma} \frac{\Delta\langle y\rangle}{g} \frac{1}{\sqrt{2 \pi} \sigma_{z}} e^{-\frac{z^{2}}{2 \sigma_{z}^{2}}} .
$$


The wakefield kick resulting from the interaction of the dipole moment of the beam distribution, with the resistive wall of the scraper with conductivity $\kappa$, is (see Appendix C):

$$
\Delta y^{\prime}(\tau)=\frac{4 r_{e} N L_{\mathrm{scr}}}{\gamma}\left(\frac{\lambda}{\pi \sigma_{z}}\right)^{1 / 2} \frac{\Delta\langle y\rangle}{g^{3}} f(\tau)
$$

where

$$
\begin{gathered}
f(\tau)=\frac{1}{\sqrt{2 \pi}} \int_{0}^{\infty} \frac{d \tau^{\prime}}{\sqrt{\tau^{\prime}}} e^{-\frac{1}{2}\left(\tau-\tau^{\prime}\right)^{2}}, \\
\lambda \equiv \frac{1}{\mu_{0} c \kappa},
\end{gathered}
$$

$L_{\text {scr }}$ is the length of the scraper, and $\tau \equiv z / \sigma_{z}$ denotes the location within the bunch. This expression assumes a smooth, cylindrically symmetric geometry of radius $g$. If the scraper consists of parallel plates separated by $2 g$ along the $y$-axis, the kick is larger by a factor of $\pi^{2} / 8,{ }^{5}$ as in the case of geometric wakefields. The above expression is valid in the range

$$
g^{2} / \lambda \gg \sigma_{z} \gg\left(\lambda g^{2}\right)^{1 / 3}
$$

For scrapers made of Ti with $\kappa \simeq 2.8 \times 10^{17} \mathrm{sec}^{-1}$, and typical bunch length for nextgeneration linear colliders of $100 \mu \mathrm{m}, \lambda=8.5 \times 10^{-11} \mathrm{~m}$ and Equation (4) is valid for all $g$ such that

$$
0.1 \mu \mathrm{m} \ll g \ll 10 \mathrm{~cm}
$$

Both conditions will be satisfied in our design. For small gaps this is the dominant wakefield effect. K. Bane has numerically integrated Equation (5) and the result is plotted in Figure 3.

In the next section we find the conditions that must be satisfied in order for mechanical collimation to be a workable collimation technique for a next-generation linear collider. Furthermore we demonstrate that mechanical collimation is precluded for the vertical degree of freedom in the Next Linear Collider (NLC), SLAC's next-generation linear collider design. In the following section we present the nonlinear collimation scheme as a possible alternative. We introduce the principle and write the conditions such a design must satisfy. These conditions determine a set of lattice parameters for the collimation systems. We present a possible lattice design, calculate its tolerances and discuss energy collimation. Before we conclude we examine the possibility of nonlinear collimation with octupoles. Finally we summarize the issues and point out the problems of the current design as well as questions remaining to be answered. 


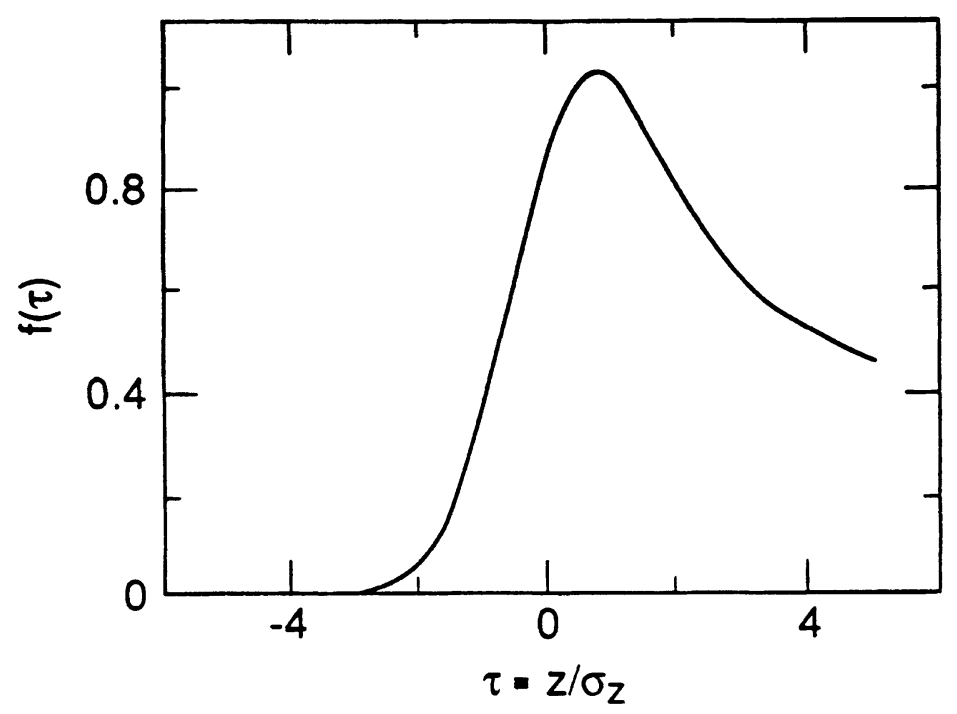

FIGURE 3: Resistive wall wakefield as a function of the longitudinal position inside the bunch.

\section{MECHANICAL COLLIMATION}

The design of a mechanical collimation section must satisfy the following requirements:

(a) The scraper half-gap must be equal to $n \sigma_{y}$,

$$
g_{y}=n \sigma_{y}
$$

for scraping particles beyond $n \sigma_{y}$.

(b) The rms value of the geometric wakefield kick must be less than $\sigma_{y}^{\prime} / 5$,

$$
\left\langle\left(\Delta y_{\mathrm{gw}}^{\prime}\right)^{2}\right\rangle^{1 / 2} \leq \frac{1}{5} \sigma_{y}^{\prime} .
$$

This requirement leads to a maximum $2 \%$ increase of the spot size. Equation (10) becomes for untapered, parallel-plate scrapers (see Appendix B),

$$
C_{1} \frac{\Delta\langle y\rangle}{g} \leq \frac{1}{5} \sigma_{y}^{\prime}
$$

where

$$
C_{1}=\frac{1}{3^{1 / 4}} \frac{\pi^{2}}{8} \frac{4 r_{e} N}{\sqrt{2 \pi} \gamma \sigma_{z}} .
$$

For $N=1 \times 10^{10}$ particles per bunch, beam energy $E=250 \mathrm{GeV}$ and $\sigma_{z}=100 \mu \mathrm{m}$, $C_{1}=0.84 \times 10^{-6}$. 
(c) The rms resistive wall wakefield kick must be less than $\sigma_{y}^{\prime} / 5$,

$$
\left\langle\left(\Delta y_{r w}^{\prime}\right)^{2}\right\rangle^{1 / 2} \leq \frac{1}{5} \sigma_{y}^{\prime}
$$

For a parallel-plate scraper this condition reads (see Appendix C),

$$
C_{2} \frac{\Delta\langle y\rangle}{g^{3}} L_{s c r} \leq \frac{1}{5} \sigma_{y}^{\prime}
$$

where

$$
C_{2}=\frac{\pi^{2}}{8}\left[\frac{K(\sqrt{3} / 2)}{2 \sqrt{\pi}}\right]^{1 / 2} \frac{4 r_{e} N}{\gamma}\left(\frac{\lambda}{\pi \sigma_{z}}\right)^{1 / 2}
$$

where $K$ is the complete elliptic integral. $C_{2}=1.12 \times 10^{-13} \mathrm{~m}$ for the parameters quoted above.

(d) As mentioned in the Introduction, in order to ensure protection of the scrapers when a mis-steered beam hits them, the beam area at the scrapers must satisfy

$$
\sigma_{x} \sigma_{y} \geq 900 \mu \mathrm{m}^{2}
$$

It is often the case that tapered scrapers must be used in order to satisfy the geometric wakefield condition (b). However tapered scrapers are necessarily longer and hence the resistive wall wakefield is increased as it varies proportionally with the length of the scraper. Therefore we must evaluate the resistive wall wake for tapered scrapers and find the common solution of both conditions (11) and (13).

To estimate the resistive wall wake of a tapered scraper we can, to a first approximation, substitute $L_{\mathrm{scr}} / g^{3}$ by the integral

$$
I=\int_{0}^{L_{\mathrm{TOT}}} \frac{d z}{g^{3}(z)}
$$

where $L_{\text {TOT }}$ is the total length of the scraper (see Figure 4), and $g(z)$ is the gap as a function of $z$.

Assuming the geometry of Figure 4 where the scraper varies linearly with the longitudinal coordinate $z$ in the regions $\mathrm{A}$ and $\mathrm{C}$, we have

$$
g(z)=\left\{\begin{array}{ll}
r+(2 / L)\left(g_{0}-r\right) z & \text { for } 0 \leq z \leq L / 2 \\
g_{0} & \text { for } L / 2 \leq z \leq L_{0}
\end{array} .\right.
$$

Then the integral $I$ is

$$
I=\frac{L\left(r+g_{0}\right)}{2 g_{0}^{2} r^{2}}+\frac{L_{0}}{g_{0}^{3}}
$$



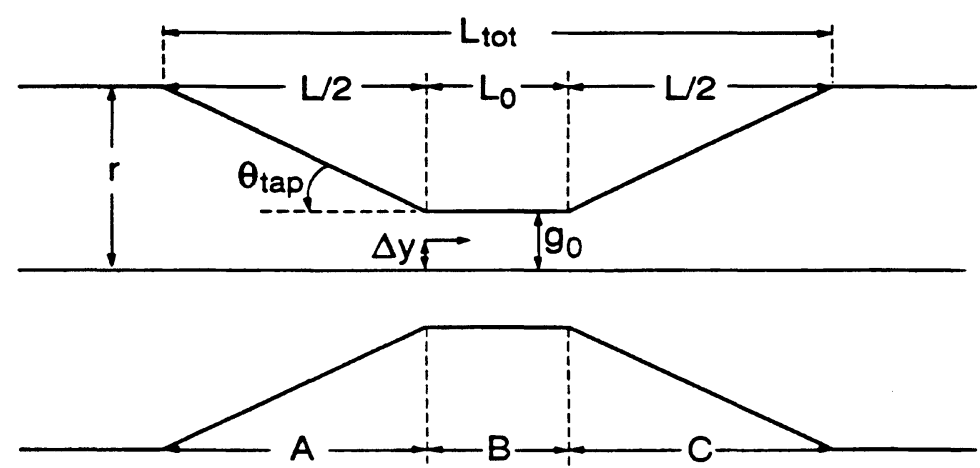

FIGURE 4: Definition of the parameters entering the calculation of tapered scrapers.

Hence the resistive wall wakefield condition, Equation (13), becomes

$$
C_{2} \Delta\langle y\rangle\left[\frac{L\left(r+g_{0}\right)}{2 g_{0}^{2} r^{2}}+\frac{L_{0}}{g_{0}^{3}}\right] \leq \frac{1}{5} \sigma_{y}^{\prime} .
$$

The geometric wakefield condition, Equation (11), becomes

$$
\frac{12 C_{1}}{\pi} \frac{\left(r-g_{0}\right)}{L} \frac{\Delta\langle y\rangle}{g_{0}} \leq \frac{1}{5} \sigma_{y}^{\prime}
$$

where we have approximated $\theta_{\text {tap }}$ by

$$
\theta_{\text {tap }} \simeq \frac{2\left(r-g_{0}\right)}{L}
$$

If we now require that the equalities of both Equations (18) and (19) be satisfied, we can eliminate $L$ and solve for $L_{0}$ in terms of $\beta_{y}$ at the scraper. For a beam offset equal to $1 \sigma_{y}$, $L_{0}$ is given by

$$
L_{0}=\frac{n^{3} \epsilon_{y}^{3 / 2}}{5 C_{2}} \beta_{\mathrm{y}, \mathrm{scr}}^{1 / 2}-\frac{30 C_{1}}{\pi} \beta_{\mathrm{y}, \mathrm{scr}}
$$

and the value of $\beta_{\mathrm{y}}$ scr that maximizes $L_{0}$ is given by

$$
\beta_{\mathrm{y}, \mathrm{scr}}=\left[\frac{\pi}{3 \times 10^{2} C_{1} C_{2}} n^{3} \epsilon_{y}^{3 / 2}\right]^{2} .
$$

We have assumed $r \gg g_{0}$. The maximum $L_{0}$ is then,

$$
L_{0, \max }=\frac{\pi}{3 \times 10^{3} C_{1} C_{2}^{2}} n^{6} \epsilon_{y}^{3}
$$


Notice the strong dependence of the last two expressions on $n$. A necessary condition for mechanical collimation to work is $L_{0, \max }$ to be of the order of a few radiation lengths of the scraper material,

$$
L_{0, \max } \geq 3 X_{0}
$$

This is because a thin scraper will not be able to disrupt the beam sufficiently in order for significant changes of the beam parameters to take place. From Equation (23) we calculated the minimum vertical normalized emittance $\epsilon_{y, N}$ for which (24) is satisfied, assuming scraping is at $10 \sigma_{y}$ and the beam energy is $250 \mathrm{GeV}: \epsilon_{y N \text {, min }}=0.75 \times 10^{-6} \mathrm{~m} \mathrm{rad}$. We also calculated the number of sigma beyond which we can scrape without violating (24), for $\epsilon_{y}=10^{-13} \mathrm{~m}$ rad: $n_{\min } \simeq 39$.

On the other hand the protection condition, Equation (14), imposes a constraint on the product of the beta functions at the scraper,

$$
\beta_{\mathrm{x}, \mathrm{scr}} \beta_{\mathrm{y}, \mathrm{scr}} \geq \frac{81 \times 10^{-20}}{\epsilon_{x} \epsilon_{y}} \mathrm{~m}^{2}
$$

Both conditions (24) and (25) must be satisfied in order for mechanical collimation to work.

Let us consider the NLC example. NLC beams are flat with a ratio of horizontal to vertical emittance equal to 100 to 1 . The incoming beam to the collimation section, which is assumed to be at the end of the linac and before the final focus, has horizontal and vertical normalized emittances equal to

$$
\epsilon_{x, N}=5 \times 10^{-6} \mathrm{~m} \mathrm{rad}, \quad \epsilon_{y, N}=5 \times 10^{-8} \mathrm{~m} \mathrm{rad} .
$$

For beam energy equal to $250 \mathrm{GeV}$, and scraping beyond $5 \sigma_{y}, L_{0, \max }=1.5 \mu \mathrm{m}$, which corresponds to about $4 \times 10^{-4}$ of a radiation length for Ti. Thus condition (24) is not satisfied, and simple mechanical collimation for the NLC vertical plane at the $5 \sigma_{y}$ level is impossible. However, it is possible for the horizontal plane.

\section{THE PRINCIPLE OF NONLINEAR COLLIMATION}

The idea here is to blow up the part of the beam we want to collimate so that mechanical scrapers can be used effectively without inducing significant wakefield kicks. Throughout this process, the core - which contributes to the luminosity of the machine - must remain unaffected.

Linear optical magnification has been excluded for the NLC as we demonstrated in the preceding section. On the other hand, higher-order multipoles such as decapoles, dodecapoles, etc., are not useful because they don't penetrate to the small distances necessary. However, for a TeV linear collider beam, skew sextupole and normal octupole fields, placed at a point where the beam size is large, seem promising. This is demonstrated in Figure 5, which displays the initial phase space of the beam distribution extended to $20 \sigma$, with $\sigma$ equal to $8 \mu \mathrm{m}$. (We have assumed $\beta=2,000 \mathrm{~m}$.) 


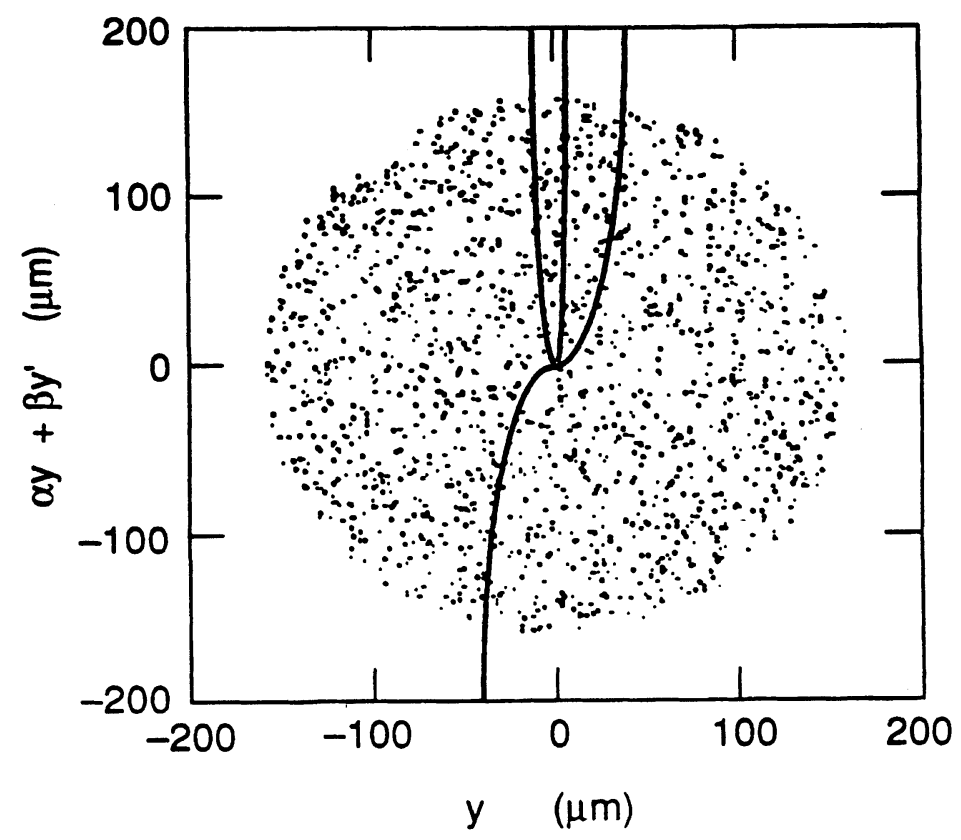

FIGURE 5: Phase space plot of a beam distribution extended to $20 \sigma$. The two curves are the angular deflections of a beam particle as functions of its position due to sextupole and octupole fields ( $\sigma=8 \mu \mathrm{m}, \beta=2,000 \mathrm{~m}$ ).

Normalized coordinates have been used so that the phase space is circular. Superimposed are plotted the angular deflections of a beam particle as functions of its transverse position due to skew sextupole and normal octupole fields, which are given by

$$
\Delta y_{\text {sext }}^{\prime}=\frac{B_{\text {pole }} L_{\text {sext }}}{a^{2}(B \rho)} y^{2}
$$

and

$$
\Delta y_{\text {oct }}^{\prime}=\frac{B_{\text {pole }} L_{\text {oct }}}{a^{3}(B \rho)} y^{3} .
$$

Notice that these expressions are highly simplified. The justification lies on the fundamental design consideration of the nonlinear collimation shceme of minimizing coupling effects at the nonlinear elements. This design requirement, which is presented later (Section 4.1.6), ensures that

$$
y^{2} \gg x^{2}
$$

at the skew sextupoles. Quantitatively, $\sigma_{y, \text { sext }}^{2} \approx 600 \sigma_{x, \text { sext }}^{2}$. The same assumption holds true for normal octupoles. Thus, the $x-y$ coupling at the nonlinear elements will be systematically neglected. 


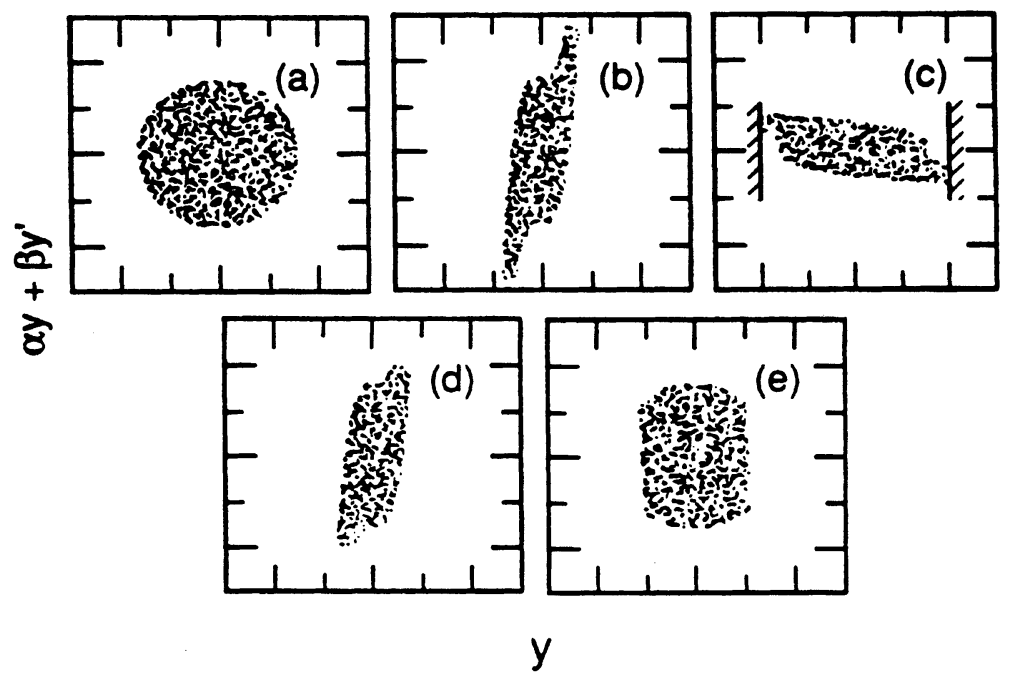

FIGURE 6: Nonlinear collimation.

In the above, $B_{\text {pole }}$ denotes the pole-tip field, $a$ denotes the pole-tip radius, $L$ denotes the length of the magnet, and $(B \rho)$ denotes the magnetic rigidity. The plot of Figure 5 uses parameters entering the above formulæ which assume their maximum values achieved by the current technology of conventional magnet construction. These values are: $1.2 \mathrm{~T}$ for the pole-tip field, $1 \mathrm{~m}$ for the magnet length, and $1 \mathrm{~mm}$ for the pole-tip radius. The energy is assumed to be $250 \mathrm{GeV}$. Notice that the sextupole field reaches deeper into the beam distribution; the octupole field does not affect the core as much as the sextupole field does. Eventually, we shall present results using both nonlinear forces. For the time being, we employ the octupole field to describe the proposed nonlinear collimation scheme.

The initial beam distribution whose phase space plot is shown in Figure 6(a) goes through an octupole magnet. The resulting phase space is shown in Figure 6(b). Then follows a rotation in betatron phase by $\pi / 2$. The new beam distribution is plotted in Figure 6(c) where the mechanical scrapers are shown by the shaded area. So by passing the beam through an octupole magnet followed by a $\pi / 2$ rotation, we have created long, angular tails which are subsequently turned into position tails and cut off by the scrapers. The core, which has been modified in the process, can be put back together by adding to the above lattice its mirror image. ${ }^{6}$ Figures 6(d) and (e) are the phase space plots of the beam distribution through this last section of the lattice. This technique is well known. A system consisting of two nonlinear elements of the same or opposite polarity (depending on their multipolarity), placed $\pi$ apart in phase advance with unit magnification, ignoring chromatic and chromo-geometric aberrations, amounts to a $-I$, where $I$ is the identity transformation.

Since in a real machine both position and angle tails cause background problems, one would like to clean up the beam profiles in both phases (say $x$ and $x^{\prime}$ ). The following schemes take this into account. It includes two lattice sections, each of which consists of two nonlinear elements $\pi$ apart; thus collimation in both phase space directions is possible. 


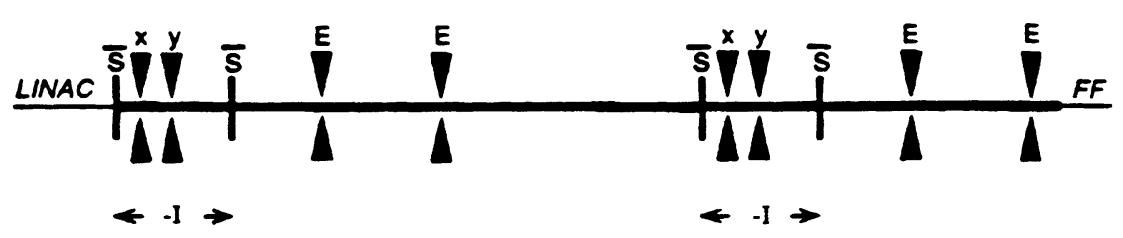

FIGURE 7: Schematic representation of the collimation systems in the NLC, located between the linac and final focus (FF). $\bar{S}$ stands for skew sextupole; $x, y, E$ stand for horizontal, vertical and energy scraper respectively.

The two lattice sections are next to each other, separated by a phase advance of $\pi / 2$. Next we demonstrate how this scheme can be used to collimate the vertical plane for the NLC.

\section{NONLINEAR COLLIMATION IN THE NLC}

\subsection{Scheme with skew sextupole pairs}

Collimation in the NLC is proposed to be done mechanically in the horizontal plane and nonlinearly in the vertical plane (scheme with skew sextupole pairs). The horizontal scrapers will be placed at high horizontal beta function points, interleaved with the vertical scrapers. Energy scraping takes place downstream from transverse scraping. A schematic representation of the collimation section of the NLC is shown in Figure 7.

The collimation design must satisfy all of the following conditions.

(a) It must scrape transverse tails beyond $n \sigma$ in both planes.

(b) It must scrape energy tails.

(c) Resistive wall wakes at all scrapers must be acceptably small.

(d) Geometric wakes at all scrapers must be acceptably small.

(e) Geometric and resistive wall wakes at the sextupoles must be acceptably small.

(f) Long sextupole aberrations must be acceptably small.

(g) Protection of horizontal, vertical and energy scrapers must be insured.

(h) Stability tolerances on sextupole and scraper offsets must be acceptable.

(i) The collimation systems must not create unacceptable chromatic or chromo-geometric aberrations.

Next we elaborate on each of the above conditions and thus arrive at the allowed design parameters of the collimation system.

4.1.1 Scraping in the vertical plane This condition implies that particles whose vertical coordinates are greater or equal to $n \sigma_{y}$ at the sextupole must be mapped into vertical positions greater or equal to $g_{y}$ at the scraper, 


$$
\Delta y_{\text {scr }}\left(\left|y_{\text {sext }}\right| \geq n \sigma_{y, \text { sext }}\right) \geq g_{y} .
$$

An $n \sigma_{y}$ particle at the skew sextupole will experience a kick

$$
\Delta y_{\text {sext }}^{\prime}=\frac{1}{2} k_{s}\left(n \sigma_{y}\right)^{2}
$$

where $k_{s}$ is the integrated sextupole strength,

$$
k_{s}=\frac{2 B_{\mathrm{pole}} L_{\mathrm{sext}}}{a^{2}(B \rho)} .
$$

Here $B_{\text {pole }}$ denotes the pole-tip field, $L_{\text {sext }}$ is the sextupole length, $a$ is the pole-tip radius of the sextupole and $B \rho$ is the magnetic rigidity. Again, the minimization of $x-y$ coupling gives rise to the above simplified expression of the skew sextupole kick. This kick will in turn give rise to an offset at the scraper

$$
\Delta y_{\mathrm{scr}}=R_{12}^{y} \Delta y_{\text {sext }}^{\prime},
$$

where $R^{y}$ is the vertical transfer matrix between sextupole and scraper. Combining the above equations we arrive at the condition

$$
\frac{1}{2} R_{12}^{y} k_{s} n^{2} \epsilon_{y} \beta_{y, \text { sext }} \geq g_{y} .
$$

4.1.2 Resistive wall wakes at the vertical scrapers

As we showed earlier the resistive wall wakefield kick at the scraper is given by

$$
\Delta y_{\mathrm{scr}}^{\prime}=C_{2} \frac{f(\tau)}{\left\langle f^{2}(\tau)\right\rangle^{1 / 2}} \frac{\Delta\left\langle y_{\mathrm{scr}}\right\rangle}{g_{y}^{3}} L
$$

which becomes at the downstream sextupole

$$
\Delta y_{\mathrm{sext}}=R_{12}^{y} \Delta y_{\mathrm{scr}}^{\prime} .
$$

An offset through the skew sextupole gives rise to a normal quadrupole kick of magnitude

$$
\Delta y_{\text {sext }}^{\prime}=\left(k_{s} \Delta y_{\text {sext }}\right) y .
$$

We require that the rms value of these kicks be less than $\sigma_{y}^{\prime} / 5$ (to avoid unacceptable longitudinal jitter of the final focal point),

$$
k_{s}\left\langle\left(\Delta y_{\text {sext }} y\right)^{2}\right\rangle^{1 / 2} \leq \frac{1}{5} \sigma_{y}^{\prime} .
$$

Condition (35), combined with eqs.(33) and (34) becomes,

$$
C_{2} k_{s} R_{12}^{y} L_{\mathrm{scr}} \beta_{y, \text { sext }} \Delta\left\langle y_{\mathrm{scr}}\right\rangle \leq \frac{1}{5} g_{y}^{3} \text {. }
$$


The jitter at the scraper can arise from two sources:

(a) The jitter in the beam emerging from the linac, which can be written in terms of the jitter in the slope at the skew sextupole,

$$
\Delta\left\langle y_{\mathrm{scr}}\right\rangle=t \sigma_{\mathrm{scr}}=t R_{12}^{y} \sqrt{\frac{\epsilon_{y}}{\beta_{y, \mathrm{sext}}}}
$$

where we assume that the jitter emerging from the linac is " $t$ " sigma. In subsequent calculations $t$ is chosen to be $1 / 5$.

(b) Jitter created by the skew sextupole kick because of beam centroid jitter at the skew sextupole. After subtracting out the average jitter, this term equals

$$
\Delta\left\langle y_{\mathrm{scr}}\right\rangle=\frac{1}{2} \sqrt{2} t^{2} k_{s} \epsilon_{y} \beta_{y, \text { sext }} R_{12}^{y}
$$

In our design the main source of jitter is (b), hence we can write condition (36) from item (b) alone,

$$
C_{2} k_{s}^{2} \epsilon_{y}\left(R_{12}^{y}\right)^{2} L_{\text {scr }} \beta_{y, \text { sext }}^{2} t^{2} \leq \frac{\sqrt{2}}{5} g_{y}^{3}
$$

Another possible source of jitter at the scraper is incoming energy jitter at the sextupoles. In our design however the vertical dispersion at the sextupoles is small, and hence this term is negligible.

4.1.3 Long sextupole aberrations The potential for long-sextupole aberrations is given by ${ }^{7,8}$

$$
V_{L S}=\frac{1}{48} k_{s}^{2} L_{\text {sext }} y^{4}
$$

assuming small horizontal beam size. Therefore the long-sextupole kick is

$$
\Delta y^{\prime}=\frac{1}{12} k_{s}^{2} L_{\text {sext }} y^{3}
$$

and we require

$$
\left\langle\left(\Delta y^{\prime}\right)^{2}\right\rangle^{1 / 2} \leq \frac{1}{5} \sigma_{y}^{\prime}
$$

This leads to the condition

$$
\frac{5 \sqrt{15}}{12} k_{s}^{2} L_{\text {sext }} \epsilon_{y} \beta_{y, \text { sext }}^{2} \leq 1 .
$$




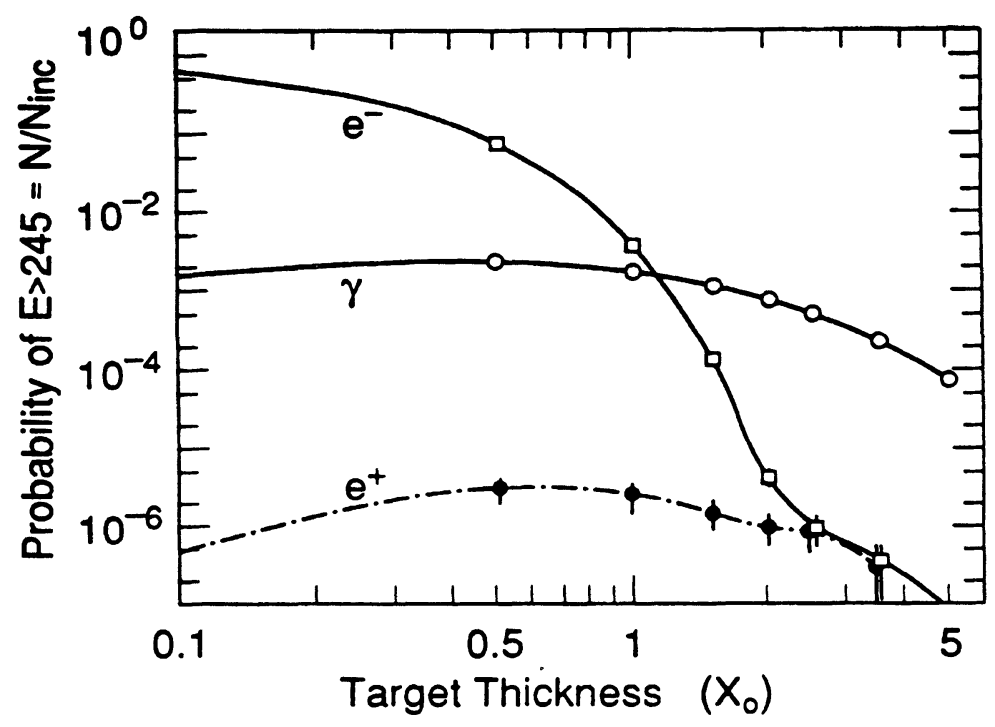

FIGURE 8: Penetration probability versus target thickness, in radiation lengths of Ti, for energies between 245 and $250 \mathrm{GeV}$.

For the two sextupoles of the $-I$ transformation, the above equation determines the maximum allowed vertical $\beta$-function,

$$
\beta_{y, \text { sext }} \leq 23,000 \mathrm{~m} .
$$

We assumed a pole-tip field of 1 Tesla, pole-tip radius of $1 \mathrm{~mm}$ and sextupole length of 10 $\mathrm{cm}$.

Equations (32), (38) and (39) determine the parameter space for the vertical plane, once the values of $R_{12}^{y}$ and $L_{\mathrm{scr}}$ are specified. The scraper length was chosen to be equal to three radiation lengths of Ti, namely $11.3 \mathrm{~cm}$. To arrive at this value we used the code EGS ${ }^{9}$ to calculate the number of electrons that make it through the $3 X_{0}$ of Ti, with energies between 245 and $250 \mathrm{GeV}$. We found that 5 out of $10^{7}$ electrons belong to this energy bin. A plot of the penetration probability versus target thickness in radiation lengths is shown in Figure 8.

If one assumes $10^{11}$ initial electrons with $1 \%$ in the tails, $10^{9}$ particles may hit the scraper. The attenuation of $5 \times 10^{-7}$ implies 50 particles have lost less than $2 \%$ of their energies. Hence another scraping section might be necessary for sufficient attenuation. Also particles hitting the scrapers near the edge may exit the scraper with little energy loss and subsequent scraping is required to remove them from the beam.

The value of $R_{12}^{y}$ is directly related to the total length of the system and hence it should be kept minimum. For $R_{12}^{y}=50 \mathrm{~m}$ (which corresponds to a length between sextupole and scraper of about $30 \mathrm{~m}$ ), an $11.3 \mathrm{~cm}$ long scraper and scraping beyond $5 \sigma_{y}$, we plotted the above equations in Figure 9. 


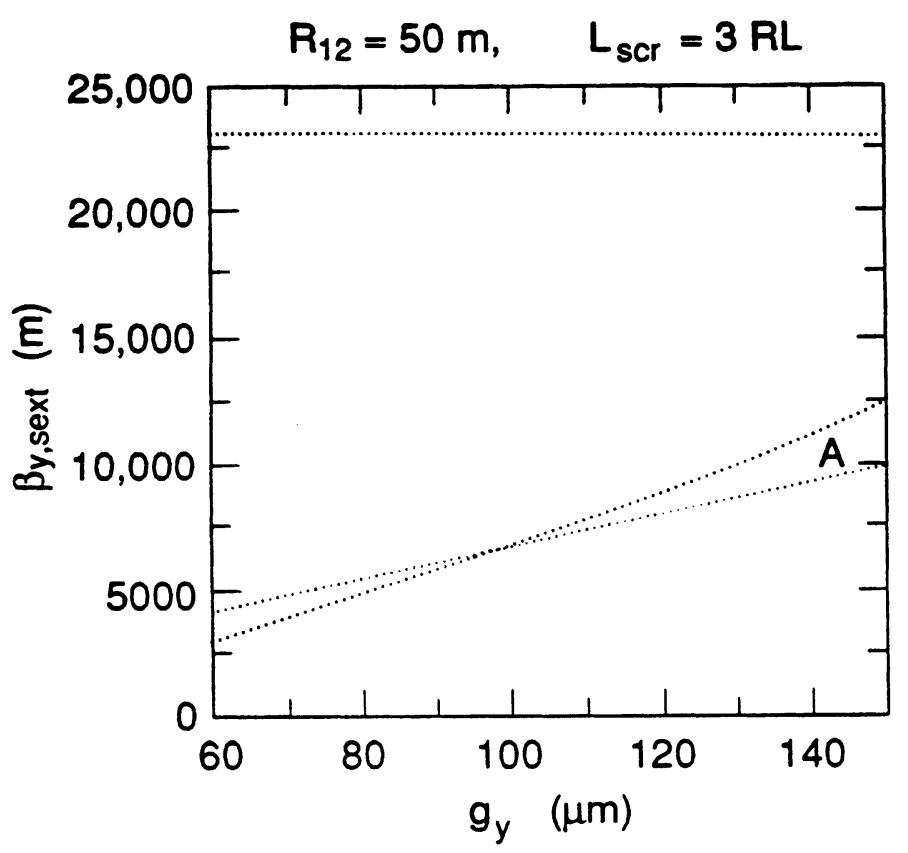

FIGURE 9: Parameter space for nonlinear collimation with sextupoles.

The region A enclosed by the three curves corresponds to the allowed space. Now we can choose the parameters of the collimation design in the vertical plane:

$$
\beta_{\mathrm{y}, \mathrm{sext}}=6,000 \mathrm{~m} \text { and } g_{y}=95 \mu \mathrm{m} .
$$

Next we check to see that the geometric wakefield condition is satisfied both at the scrapers and the sextupoles for the above choice of parameters.

4.1.4 Geometric wakefields at the vertical scrapers Following arguments similar to the ones employed before, and assuming untapered scrapers we arrive at

$$
\beta_{y, \text { sext }} \leq \frac{g_{y}^{2}}{25 C_{1}^{2} k_{s}^{2}\left(R_{12}^{y}\right)^{4} \epsilon_{y}} .
$$

This equation implies that

$$
\beta_{y, \text { sext }} \leq 14,000 \mathrm{~m}
$$

which is satisfied. condition Equation (10) at the sextupoles, for an offset 


$$
\Delta\langle y\rangle=\sigma_{y, \text { sext }},
$$

is satisfied for $\beta_{y \text {,sext }} \leq 240 \mathrm{~m}$. In our design $\beta_{y \text {,sext }}=6,000 \mathrm{~m}$ so we clearly have to taper the beam pipe at the sextupoles. In order for Equation (10) to be satisfied, the taper angle must be

$$
\theta_{\text {tap, sext }} \leq 20 \mathrm{mrad} .
$$

Each tapered section of the sextupoles is then $25 \mathrm{~cm}$ long, assuming that the beam pipe radius is $5 \mathrm{~mm}$.

To calculate the magnitude of the resistive wall wakes from the tapered sextupoles we use Equation (18) with $L_{0}=10 \mathrm{~cm}, L / 2=20 \mathrm{~cm}$ and $g_{0}=1 \mathrm{~mm}$. For $\Delta\langle y\rangle=\sigma_{y \text {,sext }}$,

$$
\left\langle\left(\Delta y_{\mathrm{rw}}^{\prime}\right)^{2}\right\rangle^{1 / 2} \simeq \frac{1}{10} \sigma_{y, \text { sext }}^{\prime},
$$

hence the resistive wall wakefield condition is satisfied at the sextupoles.

4.1.6 Horizontal considerations An important consideration that determines the $x$-plane parameters is the $x-y$ coupling at the sextupoles. To minimize coupling effects we must ensure that at the sextupoles,

$$
k_{s} y^{2} \gg k_{s} x^{2},
$$

which establishes a condition on $\beta_{x}$ at the sextupoles,

$$
\beta_{x, \text { sext }} \ll \beta_{y, \text { sext }} \frac{\epsilon_{y}}{\epsilon_{x}} .
$$

In our case,

$$
\beta_{x, \text { sext }} \ll 60 \mathrm{~m} .
$$

If we place the horizontal scrapers at a relatively high $\beta_{y}$ point, i.e., at $\beta_{y} \simeq 600 \mathrm{~m}$, then in order to ensure scraper protection, $\beta_{x}$ at this point must be greater than $1,400 \mathrm{~m}$. In fact we chose

$$
\beta_{x, \mathrm{scr}}=2,000 \mathrm{~m}
$$

which implies a scraper gap $g_{x}=700 \mu \mathrm{m}$ for $5 \sigma_{x}$ scraping. Once the $\beta$-function at the scraper is fixed, the $\beta$-function at the sextupoles follows,

$$
\beta_{x, \text { sext }}=0.1 \mathrm{~m} .
$$

The condition $k_{s} y^{2} \gg k_{s} x^{2}$ implies that the kick in the vertical plane due to a horizontal displacement is small. In addition, there is a horizontal kick proportional to $x y$. However, this kick can also be neglected, since the resulting displacement at the horizontal scraper is negligible (of the order of $0.1 \mu \mathrm{m}$ ), which is a result of the relatively small horizontal beam size at the sextupoles. 

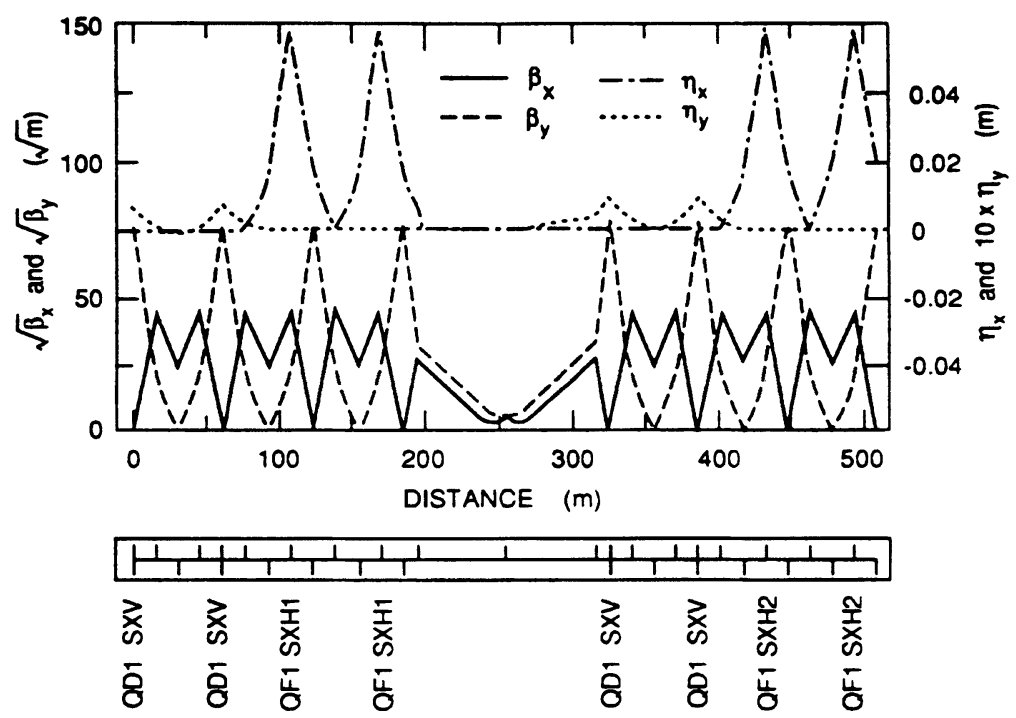

FIGURE 10: Optics design for the collimation systems in the NLC.

We now address the question of geometric and resistive wall wakefields at the horizontal scrapers. Again here both wakefield kicks must be below $\sigma_{x}^{\prime} / 5$. These conditions are simultaneously satisfied if the horizontal scrapers are tapered by an angle of $43 \mathrm{mrad}$. Each tapered section of the scrapers is then $10 \mathrm{~cm}$ long.

Finally we check to see if the horizontal geometric wakefield kick from the sextupoles is below the $\sigma_{x}^{\prime} / 5$ limit. It turns out that this condition is satisfied for

$$
\beta_{x, \text { sext }} \leq 240 \mathrm{~m},
$$

well above our design value of $0.1 \mathrm{~m}$ for $\beta_{x \text {,sext. }}$.

\subsubsection{Lattice - Energy collimation A lattice design which satisfies the above speci-} fications is presented in Figure 10.

It starts with a $-I$ transformation where horizontal and vertical scraping of the first phase space direction takes place. This is followed by a $2 \pi$ section dedicated to energy collimation. Next there is a $3 \pi / 2$ phase advance in the horizontal plane and $\pi / 2$ in the vertical plane transformer section. A phase advance of $\pi / 2$ in both planes would have been possible at the expense of considerable increase in length. The last section of the line is identical to the first one. It is used to scrape the second phase space direction and energy again. The total length of the system is about $500 \mathrm{~m}$.

Energy collimation is done by transforming off-energy particles to large amplitude ones through the introduction of horizontal dispersion. There are two scrapers in each energy scraping section placed at high dispersion points. The horizontal and vertical $\beta$-functions at these locations are the same as the ones at the horizontal scrapers. Both energy scrapers 
consist of a thin $\left(\simeq 3 X_{0}\right)$ and a thick part $\left(\simeq 20 X_{0}\right)$. The thin part will be responsible for the primary beam energy collimation. By making it thin we bypass protection problems that occur within the body of the scraper, if the whole beam is offset. The role of the thick part will be to absorb the debris from both horizontal and energy collimation that has occurred upstream. If the beam is offset, the beam area at this second scraper is much larger and thus protection of the scraper is insured.

Furthermore each of the two energy collimation sections includes a normal sextupole pair forming a $-I$ transformation. Their function is to correct the horizontal chromaticity. To correct the vertical chromaticity a small amount of vertical dispersion has been added to the lattice at the skew sextupoles. Simulations show that this entire lattice demonstrates an excellent behavior with respect to chromatic and chromo-geometric aberrations in both transverse planes.

4.1.8 Stability tolerance on scraper offset We mentioned earlier that beam jitter at the sextupoles is the main source of beam jitter at the scraper. Thus, in order to determine the stability tolerance on the scraper offset, we use Equation (37) to find

$$
y_{0, \text { scr }} \leq 0.20 \mu \mathrm{m} .
$$

From Equation (4) one can estimate an absolute steering tolerance by requiring that

$$
\left\langle\left(\Delta y_{\mathrm{rw}}^{\prime}\right)^{2}\right\rangle^{1 / 2} \leq \frac{1}{5} \sigma_{y, \mathrm{scr}}^{\prime}
$$

and solving for $\Delta\langle y\rangle$. It turns out that this tolerance is

$$
\Delta\left\langle y_{\mathrm{scr}}\right\rangle \leq 6.6 \mu \mathrm{m} .
$$

Notice that as the scrapers are moved in to place the beam must be held steady at the sextupole with a steering corrector. The above tolerance requires that the scraper motion be smooth at the $0.2 \mu \mathrm{m}$ level.

4.1.9 Stability tolerance on sextupole offsets In order to get some insight into the question of tolerances we derive a general result for the tolerance on the sextupole offset. If we combine the scraping condition Equation (32) with the requirement that the quadrupolelike kick due to the sextupole offset $y_{0, \text { sext }}$ must satisfy Equation (35),

$$
k_{s} y_{0, \text { sext }}\left\langle y^{2}\right\rangle^{1 / 2} \leq \frac{1}{5} \sigma_{y}^{\prime},
$$

we arrive at

$$
y_{0, \text { sext }} \leq \frac{n^{2}}{10} \frac{R_{12}^{y} \epsilon_{y}}{g_{y}} .
$$

Notice that the only parameters that can affect this offset tolerance are effectively the length of the system (via $R_{12}^{y}$ ), the scraper gap and the number of $\sigma$ 's we are scraping at. For our choice of parameters this jitter tolerance on sextupole motion, is 


$$
y_{0, \text { sext }} \leq 0.13 \mu \mathrm{m}
$$

4.1.10 Protection of scrapers As we mentioned in the Introduction there are two problems associated with a train of bunches hitting the scrapers: the first occurs at the surface of the scraper while the second occurs in the body of the scraper. The surface of the scrapers is protected by design. More precisely, at the horizontal scrapers the area occupied by $1 \sigma$ of the beam is

$$
\sigma_{x} \sigma_{y}=140 \mu \mathrm{m} \times 7.9 \mu \mathrm{m}=1,100 \mu \mathrm{m}^{2},
$$

close to the $900 \mu \mathrm{m}^{2}$ limit quoted earlier. Later designs will improve on this.

For the vertical scraper one can calculate the maximum particle density on the scraper for a beam which is mis-steered by more than $n \sigma_{y}$,

$$
\frac{d N}{d x d y}=\frac{N}{2 \pi n k_{s} R_{12}^{y} \epsilon_{y} \epsilon_{x}^{1 / 2} \beta_{\mathrm{y}, \text { sext }} \beta_{x, \mathrm{scr}}^{1 / 2}}
$$

where $N$ is the number of particles per train now. In order for the vertical scrapers to be protected,

$$
\frac{d N}{d x d y} \leq \frac{N}{2,000 \times 10^{-12}} \mathrm{~m}^{-2}
$$

where the more stringent limit of $2,000 \mu \mathrm{m}^{2}$ has been chosen instead of the $900 \mu \mathrm{m}^{2}$ quoted earlier. For ten bunches of $10^{10}$ particles per bunch the above criterion becomes

$$
\frac{d N}{d x d y} \leq 5 \times 10^{19} \mathrm{~m}^{-2} \text {. }
$$

In our design

$$
\frac{d N}{d x d y}=3 \times 10^{18} \mathrm{~m}^{-2}
$$

far beyond the $5 \times 10^{19} \mathrm{~m}^{-2}$ limit of Equation (41).

The problem of the body of the initial scrapers is solved by making the scrapers short, three radiation lengths of the material.

\subsection{Scheme with octupole pairs}

It is of interest to calculate the stability tolerances for octupole magnets. It turns out that for octupoles

$$
y_{0, \mathrm{oct}} \leq \frac{n^{2}}{3 \sqrt{3}} \frac{R_{12}^{y} \epsilon_{y}}{g_{y}}
$$


which leads to tolerances about a factor of 2 looser than for Equation (40) with the same choice of parameters. However, displaced octupoles give rise to sextupoles. Thus there is a need to have correctors for such aberrations, or diagnostic techniques to eliminate them. Also, the jitter tolerance at the octupoles is more than a factor of two tighter than for the sextupoles. This is because jitter at the scraper due to beam jitter at the nonlinear element varies linearly with the offset for octupoles, whereas it varies quadratically for sextupoles.

\section{CONCLUSIONS}

We have illustrated a possible collimation scheme for a $\mathrm{TeV}$ linear collider. We have precluded the possibility of using mechanical scraping for the vertical plane in the NLC design. We presented a possible alternative which employs mechanical collimation for the horizontal plane and nonlinear collimation (scheme with skew sextupole pairs) for the vertical. This design succeeds in satisfying all of the requirements imposed on collimation systems, including effective collimation of transverse and energy tails, control of wakefield effects, protection of scrapers, and control of geometric and chromatic aberrations. The stability tolerances at the scrapers and sextupoles are similar to those occurring in the NLC Final Focus system; given the precision of the beam position monitors envisioned for an NLC Final Focus, these tolerances should not rule out nonlinear collimation as a candidate for beam scraping in a future linear collider.

\section{ACKNOWLEDGEMENTS}

Interaction with K.Oide and K.Yokoya of KEK has greatly benefited our work. K. Yokoya was first to point out the importance of the resistive wall wakefield effect. Also we would like to thank K. Bane for useful comments and R. Nelson for his prompt response to our request for EGS calculations.

\section{APPENDIX A: BASIC CONCEPTS}

The transverse motion of a particle in a linear accelerator consists of betatron oscillations about a central trajectory. The transverse displacement of the particle $x_{\beta}$ from the central trajectory can be expressed as

$$
x_{\beta}=A \beta^{1 / 2}(s) \cos [\psi(s)+\delta],
$$

where $A$ and $\delta$ are constants determined by the initial conditions, and $\beta(s)$, the CourantSnyder amplitude function, ${ }^{10}$ is a periodic function of $s$, with periodicity determined by the magnetic lattice. This is a pseudoharmonic oscillation with varying amplitude $\beta^{1 / 2}(s)$, and wavelength

$$
\lambda=2 \pi \beta(s) .
$$


The phase advance $\psi(s)$ is given by

$$
\psi(s)=\int_{0}^{s} \frac{d s^{\prime}}{\beta\left(s^{\prime}\right)},
$$

and is equal to the number of oscillations in radians, per period of $s$.

Let us now consider a Gaussian distribution of particles, instead of a single particle. The rms emittance $\epsilon$ is then related to the betatron amplitude by

$$
\left(x_{\beta}\right)_{\mathrm{rms}}^{2} \equiv \sigma_{x}^{2}=\beta(s) \epsilon .
$$

In the presence of linear fields the emittance is an invariant; therefore, the betatron amplitude becomes a local measure of the beam size. For an arbitrary distribution of particles, the emittance is defined by

$$
\epsilon=\sqrt{\left\langle x^{2}\right\rangle\left\langle x^{\prime 2}\right\rangle-\left\langle x x^{\prime}\right\rangle^{2}},
$$

where the average is taken over the beam particles.

Note that throughout this paper, $\sigma$ will denote the standard deviation of the Gaussian distribution of the beam in either of the transverse dimensions $x$ or $y$. Similarly, $\sigma^{\prime}$, often referred to as the angular divergence of the beam, will denote the standard deviation of the Gaussian distribution of the beam in either of the angular dimensions $x^{\prime}$ or $y^{\prime}$.

\section{APPENDIX B: EMITTANCE GROWTH DUE TO TRANSVERSE WAKEFIELD KICKS FROM A SCRAPER}

We calculate the effect of a wakefield kick of the form

$$
\Delta y^{\prime}(z)=\frac{4 r_{e} N}{\gamma} \frac{\Delta\langle y\rangle}{g} \frac{1}{\sqrt{2 \pi} \sigma_{z}} e^{-\frac{z^{2}}{2 \sigma_{z}^{2}}}
$$

on the beam emittance. The emittance with respect to the beam centroid is defined by

$$
\epsilon^{2}=\left\langle(y-\langle y\rangle)^{2}\right\rangle\left\langle\left(y^{\prime}-\left\langle y^{\prime}\right\rangle\right)^{2}\right\rangle-\left\langle(y-\langle y\rangle)\left(y^{\prime}-\left\langle y^{\prime}\right\rangle\right)\right\rangle^{2}
$$

where $y$ and $y^{\prime}$ are the vertical displacement and slope of a particle from the central trajectory, and the average is taken over the beam distribution, along $y, y^{\prime}$ and $z$, given by

$$
f\left(y, y^{\prime}, z\right) d y d y^{\prime} d z=\frac{\beta}{(2 \pi)^{3 / 2} \sigma^{2} \sigma_{z}} e^{-\frac{\left[y^{2}+\left(\beta y^{\prime}+\alpha y\right)^{2}\right]}{2 \sigma^{2}}} e^{-\frac{z^{2}}{2 \sigma_{z}^{2}}} d y d y^{\prime} d z
$$

Under a transverse kick of the form (B.1),

$$
\langle y\rangle=0 \quad \text { and } \quad\left\langle y^{2}\right\rangle=\sigma^{2},
$$


since the kick simply displaces the beam distribution along the $y^{\prime}$ axis by a constant amount $\Delta\langle y\rangle / g$, without changing the distribution along the $y$-axis.

Furthermore,

$$
\left\langle y^{\prime}\right\rangle=\frac{\Theta_{1}}{\sqrt{2}},
$$

and

$$
\left\langle y^{\prime 2}\right\rangle=\frac{\sigma^{2}}{\beta^{2}}\left(1+\alpha^{2}\right)+\frac{\Theta_{1}^{2}}{\sqrt{3}},
$$

where

$$
\Theta_{1} \equiv \frac{4 r_{e} N}{\sqrt{2 \pi} \sigma_{z} \gamma} \frac{\Delta\langle y\rangle}{g} .
$$

Also,

$$
\left\langle y y^{\prime}\right\rangle=-\frac{\sigma^{2} \alpha}{\beta} .
$$

Thus the total emittance is given by

$$
\epsilon^{2}=\epsilon_{0}^{2}\left[1+\frac{(2-\sqrt{3})}{2 \sqrt{3}}\left(\frac{\Theta_{1}}{\sigma^{\prime}}\right)^{2}\right],
$$

where $\epsilon_{0}=\sigma^{2} / \beta$ and $\sigma^{\prime 2}=\epsilon_{0} / \beta$.

\section{APPENDIX C: EMITTANCE GROWTH DUE TO RESISTIVE WALL WAKEFIELD}

\section{C1 Resistive wall wakefield}

The transvere wake force experienced by a test particle $e$, as a result of the interaction of the dipole moment $I_{1}$ of the beam distribution with the resistive wall of a smooth, cylindrically symmetric pipe of radius $g$ and conductivity $\kappa$ is, ${ }^{11}$

$$
F_{\perp}=\frac{2 e I_{1}}{\pi g^{3}}\left(\frac{c}{\kappa}\right)^{1 / 2} \frac{1}{Z^{1 / 2}},
$$

where

$$
I_{1}=\frac{q}{\sqrt{4 \pi \epsilon_{0}}} \Delta\langle y\rangle .
$$

Upper case $Z$ designates the longitudinal separation between the test charge and the beam, i.e., $Z=c t-z$, where $z$ is the longitudinal coordinate for the test particle. This expression is valid in the range 


$$
\frac{g^{2}}{\lambda} \gg Z \gg\left(\lambda g^{2}\right)^{1 / 3}
$$

where $\lambda$ is defined as

$$
\lambda \equiv \frac{1}{\mu_{0} c \kappa} .
$$

To calculate the total force on $e$ at point $z$ within a Gaussian bunch, we integrate over the appropriate distribution ahead of $z$. For $Z$ near $\left(\lambda g^{2}\right)^{1 / 3}$, the exact wake force has a maximum and then goes to zero at $Z=0$. However, since $\left(\lambda g^{2}\right)^{1 / 3} \ll \sigma_{z}$, even if we replace the correct wake by continuing the expression (1.1) in this region, we make a negligible error. Thus the total force is given by

$$
F_{e}(z)=\frac{e^{2} N}{\sqrt{2 \pi} \sigma_{z}} \frac{c Z_{0}}{\pi}\left(\frac{\lambda}{\pi}\right)^{1 / 2} \frac{\Delta\langle y\rangle}{g^{3}} \int_{z}^{\infty} \frac{d z^{\prime}}{\left(z^{\prime}-z\right)^{1 / 2}} e^{-\frac{z^{\prime 2}}{2 \sigma_{z}^{2}}}
$$

and the transverse kick is given by

$$
\Delta y^{\prime}(z)=\frac{F_{e}(z) L}{m \gamma c^{2}},
$$

where $L$ is the length of the finite conductivity element, e.g., the scraper.

Thus, the wakefield kick is given by

$$
\Delta y^{\prime}(\tau)=\frac{4 r_{e} N L}{\gamma}\left(\frac{\lambda}{\pi \sigma_{z}}\right)^{1 / 2} \frac{\Delta\langle y\rangle}{g^{3}} \frac{1}{\sqrt{2 \pi}} \int_{0}^{\infty} \frac{d \tau^{\prime}}{\sqrt{\tau^{\prime}}} e^{\frac{-\left(\tau-\tau^{\prime}\right)^{2}}{2}},
$$

where $\tau=z / \sigma_{z}$ denotes the location within the bunch.

\section{C2 Emittance growth}

Now we calculate the effect of this wakefield kick on the beam emittance. The emittance with respect to the beam centroid is given by (B.2) and the average over the beam distribution yields

$$
\begin{gathered}
\left\langle y^{\prime}\right\rangle=\Theta_{2} \frac{\Gamma(1 / 4)}{2 \sqrt{2 \pi}}, \\
\left\langle y^{\prime 2}\right\rangle=\frac{\sigma^{2}}{\beta^{2}}\left(1+\alpha^{2}\right)+\Theta_{2}^{2} \frac{K(\sqrt{3} / 2)}{2 \sqrt{\pi}}, \\
\left\langle y y^{\prime}\right\rangle=-\frac{\sigma^{2} \alpha}{\beta},
\end{gathered}
$$


where $K$ is the complete elliptic integral and $\Theta_{2}$ is defined by

$$
\Theta_{2} \equiv \frac{4 r_{e} N L}{\gamma}\left(\frac{\lambda}{\pi \sigma_{z}}\right)^{1 / 2} \frac{\Delta\langle y\rangle}{g^{3}}
$$

The total emittance is given by

$$
\epsilon^{2}=\epsilon_{0}^{2}\left\{1+\frac{\Theta_{2}^{2}}{2 \sqrt{\pi} \sigma^{\prime 2}}\left[K(\sqrt{3} / 2)-\frac{\Gamma^{2}(1 / 4)}{4 \sqrt{\pi}}\right]\right\},
$$

where $\sigma^{\prime 2}=\epsilon_{0} / \beta$.

\section{REFERENCES}

1. D. Walz, private communication.

2. K.L.F. Bane and P.L.Morton, "Deflection by the Image Current and Charges of a Beam Scraper," SLACPUB-3983 (1986).

3. K.L.F. Bane, private communication.

4. K. Yokoya, "Impedance of Slowly Tapered Structures," CERN SL/90-88(AP) (1990).

5. H. Henke and O. Napoly, Wakefields Between Two Parallel Resistive Plates, EPAC '90 Proc. (1990) p.1046.

6. The original idea of using the $-I$ transformation with the scraper in the middle came from $\mathrm{K}$. Oide of KEK.

7. J. Irwin, "The Application of Lie Algebra Techniques to Beam Transport Design," SLAC-PUB-5315 (1990).

8. K. Oide, "Design of Optics for the Final Focus Test Beam at SLAC," SLAC-PUB-4953 (1989).

9. W.R. Nelson, H. Hirayama and D.W.O. Rogers, SLAC-265 (1985).

10. E.D. Courant and H.S. Snyder, "Theory of the Alternating-Gradient Synchrotron," Ann. of Phys., 3 (1958) 1.

11. A.W. Chao, "Coherent Instabilities of a Relativistic Bunched Beam," SLAC-PUB-2946 (1982). 